\title{
Outcome of Management of Pseudomyxoma Peritonei: National Cancer Institute Experience
}

\author{
Z. Gad1', 0. Nassar1', H. Soliman'1 ${ }^{*}$, S. Mohamed ${ }^{2}$, M. Mohamed ${ }^{3}$ \\ ${ }^{1}$ Surgical Oncology Department, National Cancer Institute, Cairo University, Giza, Egypt \\ ${ }^{2}$ Medical Oncology Department, National Cancer Institute, Cairo University, Giza, Egypt \\ ${ }^{3}$ Ain Shams University, Cairo, Egypt \\ Email: *soliman@daad-alumni.de
}

How to cite this paper: Gad, Z., Nassar, O., Soliman, H., Mohamed, S. and Mohamed, M. (2018) Outcome of Management of Pseudomyxoma Peritonei: National Cancer Institute Experience. Journal of Cancer Therapy, 9, 323-337.

https://doi.org/10.4236/jct.2018.94030

Received: March 17, 2018

Accepted: April 24, 2018

Published: April 27, 2018

Copyright $\odot 2018$ by authors and Scientific Research Publishing Inc. This work is licensed under the Creative Commons Attribution International License (CC BY 4.0).

http://creativecommons.org/licenses/by/4.0/

\begin{abstract}
Background: The current treatment for pseudomyxoma peritonei (PMP) consists of radical cytoreductive surgery (CRS) followed by hyperthermic intra-peritoneal chemotherapy (HIPEC). Aim: To assess PMP patients regarding the clinical and pathological characteristics, the treatment including surgery (CRS) and chemotherapy either HIPEC type or post-operative systemic chemotherapy aiming to evaluate end results regarding recurrence and survival. Patients and Methods: This retrospective study included 39 patients with PMP who were diagnosed, treated and followed-up from 2009-2014 at National Cancer Institute, Cairo, Egypt. Results: High grade mucinous adenocarcinoma was found in $23.1 \%$ of patients. Patients with low grade tumor showed higher survival rate compared with patients with high grade disease. The mean operative PCI score (peritoneal cancer index) that was done to all patients who were explored was 15.81. Our study reported success to achieve complete cytoreduction that was combined with HIPEC in $44 \%$ of patients who were planned for this modality. Treatment related postoperative grade (3 - 5) complications mainly surgery related developed in 17.3 of patients. Operative mortality was $22.2 \%$. The follow up period in our study was quite short (mean 22.9). However the overall survival at the end of the follow up in our study was $48.7 \%, 1$ year survival was $82 \%$, and 2 year survival was $41 \%$. The overall survival in patients treated with CRS and HIPEC was $66.6 \%$, with 1 year and 2 year survival of $91 \%$ and $66.6 \%$ respectively. Only 2 patients developed recurrent disease during the follow up period. Conclusions: The outcome of PMP treatment process is extremely variable. Combined CRS and HIPEC is considered the best therapeutic approach for patients with PMP. Surgical experience combined with proper patient selection have to be built up together to improve the outcome. That could only be achieved through more centralization of patients' treatment in specialized units or center.
\end{abstract}




\section{Keywords}

HIPEC, NCI Experience, Pseudomyxoma Peritonii

\section{Introduction}

Pseudomyxoma peritonei (PMP) is a rare intraperitoneal tumor, characterized by disseminated intraperitoneal tumor implants on peritoneal surfaces. The disseminated neoplastic cells produce mucin, which leads to the characteristic mucinous ascites [1].

Traditionally PMP has been treated with serial debulking procedures. This technique can provide relief for some time; however repeated debulking procedures become increasingly difficult, and lead to more complications [2]. A more aggressive approach combining Cytoreductive Surgery (CRS) and Intraperitoneal Chemotherapy (IPEC) is being used. CRS is performed to remove as much macroscopic disease as possible often undertaken according to Sugarbaker's protocol. This is combined with IPEC that targets the microscopic residual disease and free neoplastic cells in the peritoneal cavity. This chemotherapy may be heated (hyperthermic) (HIPEC), which is used intra-operatively; or this can be used either preoperatively or early postoperative [3]. Retrospective studies have shown that debulking procedures alone can provide 5 -year survival rates of $55 \%$ - 75\%, however, around $90 \%$ of patients have disease recurrence within 2 years and will often require multiple debulking procedures [4].

Combining CRS and HIPEC can greatly improve survival with average 5 year survival rate around 76\% [5]. However other studies have shown that 5 year disease free survival can range from $37.4 \%$ to $74 \%$. This difference between studies may be a reflection of patient selection and experience of the centers than an evaluation of the treatment regimen [6].

The aim of this work was to assess patients with PMP regarding the clinical and pathological characteristics, the treatment including surgery (CRS) and chemotherapy either HIPEC type or post-operative systemic chemotherapy aiming to evaluate end results regarding recurrence and survival.

\section{Patients and Methods}

This is a retrospective study that was conducted at National Cancer Institute (NCI) in Cairo, Egypt. The study included patients with PMP who were diagnosed (radiologically and pathologically), treated and followed up in the period from 2009 to 2014.

\section{Methods}

Histologically proven PMP were identified at the histopathology department database. Patients' files were retrieved from the biostatistics department. Data were extracted from the medical records, and selected variables were collected 
including; demographic characteristics included age and gender, patient medical history regarding diabetes mellitus and hypertension, history and number of previous surgeries that were related to PMP diagnosis and management, time between disease diagnosis and definitive surgery, number and types of lines of preoperative and postoperative systemic chemotherapy, and patients' response, pathological features of the disease including type, grade, margins and lymph nodes involvement, preoperative evaluation including laboratory, radiological investigations including CT scan for the chest and abdomen, MRI, and PET CT if available, to estimate the preoperative PCI and metastasis, presence of extra-peritoneal metastasis, operative data (operative PCI score, whether complete or incomplete cytoreduction was done, data about hyperthermic intra peritoneal chemotherapy and type of chemotherapy, intraoperative morbidity, operative mortality), post-operative data (ICU admission and duration of ICU admission, hospital stay was calculated starting from date of operation till date of discharge, follow up data including disease progression and recurrence), survival data (event free survival was calculated from date of surgery till date of recurrence, progression or date of last follow up, overall survival was calculated from date of diagnosis of the primary cancer till date of death or date of last follow up if not died).

Date of diagnosis was determined by the date of the operation or by the date of the first pathology report if biopsy was taken. Histopathology of the tumor was classified according to the WHO classification, to low grade mucinous carcinoma and high grade mucinous carcinoma.

Tumor markers included in the study were CA125, CA19-9 and CEA. Data about completeness of cytoreduction was not documented according the CC score, so it was estimated according to R score; where R0 means no microscopic residuals at the end of the operation, R2 means macroscopic residual, while R1 means microscopic residuals detected as positive margin. Operative complications were considered as any deviation from the ideal operative and postoperative course, while operative mortality was defined as death within one month of the operation.

Progression and recurrence was diagnosed in case of marked rise of tumor markers and/or evidence of relapse in abdominal CT scan, or during laparotomy for any cause.

\section{Results}

This study included 39 patients with pseudomyxoma peritonei. Their mean age was 53.8 years (Table 1). Female to male ratio was about 1:1 [20 females (51\%): 19 males (49\%)]. Diabetic patients represented $5.6 \%$ of patients and $12.5 \%$ of patients were hypertensive. Twenty eight patients $(71.7 \%)$ were diagnosed outside NCI, while 11 patients (28.2\%) were diagnosed at NCI. The circumstances leading to the discovery of PMP were; open biopsy during abdominal surgery in $84.6 \%(n=33)$ of patients, core biopsy in $10.2 \%(n=4)$, laparoscopic in $2.6 \%$ (one patient), and cytology in $2.6 \%$ (one patient). These abdominal surgeries included 
Table 1. Descriptive statistics.

\begin{tabular}{ccc}
\hline \multicolumn{2}{c}{ Descriptive Statistics } & \\
\hline Age (years) & Range & Mean \pm SD \\
\hline $\begin{array}{c}\text { Time between diagnosis } \\
\text { and operation (months) }\end{array}$ & $25-70$ & $53.846 \pm 12.014$ \\
ICU stay (days) & $1-15$ & $4.577 \pm 3.880$ \\
Total hospital stay (days) & $1-21$ & $6.368 \pm 5.387$ \\
Follow up time (months) & $1-68$ & $11.115 \pm 13.698$ \\
\hline
\end{tabular}

panhystrectomy in 11 patients (33.3\%), exploration in 13 patients (39.3\%), appendectomy in 4 patients (30.3\%), hernioplasty in 3 patients $(9.1 \%)$, during caesarian section in one patient (3\%), and iliopsoas mass in one patient (3\%). Twenty three patients (58.9\%) presented with abdominal or pelvic mass, 6 patients with abdominal or pelvic cysts while ascites was manifested in $66.7 \%$ of patients ranging from mild to massive. Extra-peritoneal metastasis was reported in 4 patients (10.2\%) that included liver metastasis in 3 patients and one patient presented with spread along the insertion of iliopsoas muscle at the upper thigh.

CEA was elevated in $15 / 22$ patients (68.1\%) in whom the test was done while CA 19-9 was measured in 19 patients and it was elevated in 9 patients (47\%). CA125 was measured in 14 patients and it was elevated in 6 patients (42\%). Low grade mucinous adenocarcinoma was reported in 30 patients $(76.9 \%)$, while high grade mucinous adenocarcinoma in 9 patients $(23.1 \%)$.

In preoperative assessment, 32 patients $(82 \%)$ had history of one previous surgery related to their illness, while $12 \%$ did not undergo any previous surgeries. The mean time between disease diagnosis and the operation was 4.6 months (range $=1-15)$. Twenty six patients $(68 \%)$ were eligible and planned for combined CRS and HIPEC, and one patient for laparoscopic exploration. Only 12 patients (44.4\%) underwent cytoreductive surgery and HIPEC, 6 patients were inoperable. Five patients had CRS without HIPEC; as their operations were aborted after completion of cytoreduction due to intraoperative complications or anesthetic limitations. However the operation was incomplete in 3 patients, and aborted before complete cytoreduction due to intraoperative morbidity. The mean operative PCI score was 15.8 (Range $=0$ - 31).

The other 11 patients (28\%) who were excluded from CRS and HIPEC were planned for follow up with or without systemic chemotherapy, or for best supportive care. One patient died before treatment. Among patients that underwent CRS with or without HIPEC, a macroscopic complete cytoreduction (R0) was reached in 13 patients (76.4\%), R1 in 2 patients, and R2 in 2 patients. Bowel resection was done in $57.6 \%$ of patients who were planned for surgery.

During the study period, 8 patients $(20.5 \%)$ received one line of systemic chemotherapy, 5 patients $(12.8 \%)$ received two lines. The response of the disease to chemotherapy was progressive in 5 patients and stationary in the other $8 \mathrm{pa}-$ 
tients. The different lines of chemotherapy used in those patients are shown in Table 2. However three patients gave history of treatment with chemotherapy outside NCI but the lines used were not documented.

\subsection{Morbidity and Mortality}

Intraoperative complications encountered in 8 patients (29\%), the most common was bleeding in 6 patients. Treatment related postoperative grade (3 - 5) complications mainly surgery related developed in 4 patients $(17.3 \%)$ that consisted of leakage in 2 patients, pulmonary embolism, and reactionary hemorrhage. Operative mortality was $22.2 \%(n=6)$. Nineteen patients $(70.4 \%)$ needed postoperative ICU admission with the mean ICU stay was 6.3 days (range 1 21 ), while the mean postoperative hospital stay was 11.1 days (range 1 - 68).

\subsection{Outcome and Survival}

The mean follow up period was 22.9 months (range $1-63$ ). At the end of the follow up 19 patients were alive and 20 patients (51.3\%) died, the overall survival was $48.7 \%$, 1 year overall survival rate was $82 \%$, and 2 year overall survival rate was $41 \%$. The 1 and 2 years overall survival in patients treated with CRS and HIPEC were $91 \%$, and $66.6 \%$ respectively. Two patients (16.7\%) developed disease recurrence that needed reoperation with cytoreductive surgery and HIPEC.

There was a significant relation between patients' age and their final status or survival; young patients were associated with higher survival rates. In patients who underwent the operation after a period less than 6 months survival rate was $45 \%, 66.7 \%$ in operations within $6-12$ months, and $0 \%$ in operations done within a period more than a year. However these results were insignificant. Overall survival in patients with low grade mucinous adenocarcinoma was $56.6 \%$ compared to $22.2 \%$ in patients with high grade mucinous adenocarcinoma but the difference was of borderline significance $(\mathrm{P}=0.070)$. Patients with low grade tumor who underwent CRS and HIPEC showed significantly better overall survival when compared with those with high grade disease who underwent the same surgery $(\mathrm{P}=0.028)$. Correlating the number of elevated preoperative tumor markers and survival showed no significant relation between the number of elevated tumor markers and the overall survival. In all patients who were explored the mean operative PCI score was 15.81 (range 1 - 30). The mean PCI score of those patients who were alive at the end of the follow up period was 13, compared to 18 in patients who died. Although the mean PCI score was higher in patients who died at the end of follow up period, the correlation between the mean PCI score and survival or recurrence in our study was not significant (Table 3). Among patients who had any trial for CRS and HIPEC, operative complications were significantly related to post-operative mortality (Table 4), and overall survival (Table 3). Survival was $12.5 \%$ in patients who developed operative complications compared with $63.2 \%$ among other patients. There was no significant relation between treatment with chemotherapy and the overall survival (Table 3). 
Table 2. Lines of chemotherapy used.

\begin{tabular}{cc}
\hline Chemotherapy line & Number of patients \\
\hline Taxol-Carboplatin & 6 \\
FOLFOX & 2 \\
Taxol-Gemzar & 1 \\
Cisplatin & 1 \\
FU-xeloda & 1 \\
Xeloda & 1 \\
Oxaloplatin-xeloda & 2 \\
IFL & 1 \\
\hline
\end{tabular}

Table 3. Effect of different variables done on the survival rate.

\begin{tabular}{|c|c|c|c|c|c|c|}
\hline & & Alive & & Death & $t / x^{2}$ & $P$ value \\
\hline Operation Done & $\mathbf{N}$ & $\%$ & $\mathrm{~N}$ & $\%$ & & \\
\hline CRS & 1 & 20.00 & 4 & 80.00 & & \\
\hline CRS + HIPEC & 8 & 66.67 & 4 & 33.33 & & \\
\hline Inoperable & 3 & 50.00 & 3 & 50.00 & 7.106 & 0.130 \\
\hline aborted & 0 & 0.00 & 3 & 100.00 & & \\
\hline Lap. Exploration & 1 & 100.00 & 0 & 0.00 & & \\
\hline Age (Range, Mean \pm SD) & & $\begin{array}{c}25-61 \\
47.42 \pm 10.95\end{array}$ & & $\begin{array}{c}45-70 \\
59.95 \pm 9.73\end{array}$ & -3.782 & $0.001^{\star}$ \\
\hline Time Interval (N, \%) & $\mathbf{N}$ & $\%$ & $\mathrm{~N}$ & $\%$ & & \\
\hline 1 to 6 & 9 & 45.00 & 11 & 55.00 & 2.205 & 0.332 \\
\hline 6 to 12 & 2 & 66.67 & 1 & 33.33 & & \\
\hline$>12$ & 0 & 0.00 & 2 & 100.00 & & \\
\hline Path Grade (N, \%) & $\mathbf{N}$ & $\%$ & $\mathrm{~N}$ & $\%$ & & \\
\hline Low & 17 & 56.67 & 13 & 43.33 & 3.288 & 0.070 \\
\hline High & 2 & 22.22 & 7 & 77.78 & & \\
\hline Total & 19 & 48.72 & 20 & 51.28 & & \\
\hline Operation + grade $(\mathrm{N}, \%)$ & $\mathbf{N}$ & $\%$ & $\mathbf{N}$ & $\%$ & & \\
\hline $\begin{array}{l}\text { CRS + HIPEC with } \\
\text { Low grade tumor }\end{array}$ & 8 & 80.00 & 2 & 20.00 & 4.800 & $0.028^{*}$ \\
\hline $\begin{array}{l}\text { CRS + HIPEC with } \\
\text { High grate tumor }\end{array}$ & 0 & 0.00 & 2 & 100.00 & & \\
\hline $\begin{array}{c}\text { Number of } \\
\text { elevated markers }(\mathrm{N}, \%)\end{array}$ & $\mathbf{N}$ & $\%$ & $\mathrm{~N}$ & $\%$ & & \\
\hline No & 4 & 57.14 & 3 & 42.86 & 0.152 & 0.985 \\
\hline One & 5 & 62.50 & 3 & 37.50 & & \\
\hline Two & 4 & 66.67 & 2 & 33.33 & & \\
\hline Three & 2 & 66.67 & 1 & 33.33 & & \\
\hline Total & 15 & 62.50 & 9 & 37.50 & & \\
\hline
\end{tabular}




\section{Continued}

\begin{tabular}{|c|c|c|c|c|c|c|}
\hline OP. PCI & & & & & & \\
\hline Range, & & $1-31$ & & $10-30$ & & \\
\hline Mean \pm SD & & $13 \pm 9.76$ & & $18.43 \pm 5.50$ & & \\
\hline $\begin{array}{c}\text { Operative } \\
\text { Complications (N, \%) }\end{array}$ & $\mathbf{N}$ & $\%$ & $\mathbf{N}$ & $\%$ & & \\
\hline No & 12 & 63.16 & 7 & 36.84 & 5.787 & $0.016^{*}$ \\
\hline Yes & 1 & 12.50 & 7 & 87.50 & & \\
\hline Previous lines of CTH (N, \%) & $\mathbf{N}$ & $\%$ & $\mathbf{N}$ & $\%$ & & \\
\hline No & 12 & 46.15 & 14 & 53.85 & 0.205 & 0.651 \\
\hline Yes & 7 & 53.85 & 6 & 46.15 & & \\
\hline
\end{tabular}

Table 4. Effect of operative complications on operative mortality rate.

\begin{tabular}{|c|c|c|c|c|c|c|c|c|}
\hline \multirow{3}{*}{$\begin{array}{c}\text { Operative } \\
\text { complications }\end{array}$} & \multicolumn{6}{|c|}{ Op Mortality } & \multirow{2}{*}{\multicolumn{2}{|c|}{ Chi-Square }} \\
\hline & \multicolumn{2}{|c|}{ No } & \multicolumn{2}{|c|}{ Yes } & \multicolumn{2}{|c|}{ Total } & & \\
\hline & $\mathbf{N}$ & $\%$ & $\mathbf{N}$ & $\%$ & $\mathbf{N}$ & $\%$ & $\mathrm{X}^{2}$ & P-value \\
\hline No & 17 & 89.47 & 2 & 10.53 & 19 & 100.00 & & \\
\hline Yes & 4 & 50.00 & 4 & 50.00 & 8 & 100.00 & 5.075 & $0.024^{*}$ \\
\hline Total & 21 & 77.78 & 6 & 22.22 & 27 & 100.00 & & \\
\hline
\end{tabular}

\section{Discussion}

The introduction of cytoreductive surgery followed by intraperitoneal chemotherapy as a more aggressive approach has resulted in a favorable impact on survival in patients with PMP. The pathological process of PMP is a subclinical event in the majority of patients, and pathological diagnosis is commonly made after laparotomy. Most of patients included in our study were diagnosed outside NCI, and were referred with at least one previous laparotomy. PMP is generally 2 - 3 times more common in females. Chua et al. [7] reported that age more than 53 years negatively affects overall survival. Our study reported a male to female ratio of 1:1 with mean age 53.8 years. We reported a significant relation between patients' age and their final status or survival, as young patients were associated with higher survival rates.

Improving the outcome of surgery requires careful patient selection through the proper preoperative investigations. However accurate assessment of preoperative PCI is not often possible. The surgical PCI score is considered a strong prognostic factor, as it helps to predict the chance to achieve complete cytoreduction, which directly affects patients' morbidity and survival [8]. In this study the mean operative PCI score was 15.81. Although the mean PCI score was higher in patients who died at the end of follow up period, the correlation between the mean PCI score and survival or recurrence in our study was not significant. 
Most of patients with PMP have elevation in one or more of the serum tumor markers. However it is non-specific to diagnose PMP but it is considered significant and independent prognostic factor regarding disease free survival and overall survival. A 5 year overall survival of $91 \%$ was reported in patients with no elevation in preoperative tumor markers compared to $60.8 \%$ overall survival in patients with elevated tumor markers [9]. In the current work, the correlation between higher tumor markers and the final status of patients was insignificant. This might be related to small number of patients who were tested for tumor markers.

The most recent pathological classification of PMP is according to the WHO classification, categorized the disease to low grade and high grade mucinous adenocarcinoma [10]. So in this study it was important to unify the classification depending on the grade and cell type. We reported low grade mucinous adenocarcinoma in $76.9 \%$ of patients. Patients with low grade tumor showed higher survival rate compared with patients with high grade disease. However these results were not significant.

The histopathological grade is considered an important prognostic factor regarding patients' outcome, as patients with high grade tumor demonstrated invasive component and had poor outcome compared with those with low grade disease.

Survival was much better with low grade tumor than those with high grade tumor. So a non-invasive histopathology is extremely important in selecting patients who are most likely to benefit from this treatment strategy [11]. In patients with low grade tumor the overall survival rate at 20 years was $68 \%$ versus $18 \%$ in patients with high grade. Low grade patients with a PCI score less than 20 had 20 years survival of $90 \%$, versus $65 \%$ survival among those with PCI more than 20 with the same pathology. High grade tumor patients with PCI less than 20 the 10 years survival was $45 \%$ compared to $28 \%$ in patients with PCI more than 20 [12]. However we reported higher survival in patients with low grade tumors compared to high grade, it was difficult to compare our results with other studies due to the short follow up period in this study.

The pathological process is mostly subclinical, and may be an incidental finding during laparotomy. Symptoms of appendicitis, increased abdominal girth, and ovarian mass are the most common presentation. Abdominal pain, hernia and ascites are less common presentations [13]. In this study $58.9 \%$ of patients presented with abdominal or pelvic mass, 6 patients with abdominal or pelvic cysts while ascites was manifested in $66.7 \%$ of patients ranging from mild to massive. Extra-peritoneal metastasis was reported in $10 \%$.

To identify appropriate surgical candidates and to predict tumor resectability, it was extremely important to do preoperative imaging. A full contrast abdomino-pelvic CT can provide information about the extent of the disease and to estimate the radiological PCI. CT sensitivity varies from $60 \%$ to $90 \%$, depending on the quality of CT, size of tumor nodules, the regions examined and the interpretation of the radiologist. However an accurate assessment of radiological PCI 
is often not possible as it is poor at detecting nodules less than $5 \mathrm{~mm}$ so the presence of milliary disease is a common cause of underestimation of PCI [14]. Recent advances in MRI suggested that it can be a better predictor of the surgical PCI compared to CT especially in detection of small bowel involvement. However it is limited by the experience of the radiologist [15].

Extent of the disease reported with PCI score is the most important factor in predicting resectability and hence there is a strong relation between the PCI and the chance to achieve complete cytoreduction that is directly related to survival. Studies reported that when PCI score is greater than 20 the 5 year survival rate is less than $10 \%$ and here it is recommended to avoid cytoreductive surgery and HIPEC in PCI score more than 20 [8].

The most frequently used regimens in HIPEC are; Mitomycin C/doxorubicin, perfused intraperitoneal for 90 minutes (Sugarbaker's protocol) [12], and Oxaloplatin perfused intraperitoneal for 30 minutes (Elias' protocol) [16]. However there are many other regimens that may include single chemotherapeutic drug Mitomycin C or Oxaloplatin alone.

This study reported success to achieve complete cytoreduction that was combined with HIPEC in only $44 \%$ of patients who were planned for this treatment modality. The chemotherapeutic regimen we used was Mitomycin $\mathrm{C}$ alone heated to $42^{\circ} \mathrm{C}$ and perfused through closed abdomen technique.

In a series of patients, Smeenk et al. [6] reported complete cytoreduction in $32 \%$ of patients. However, Jarvinen et al. [17] in another series reported complete cytoreduction combined with HIPEC in $81 \%$ of patients who were suitable for CRS and HIPEC. Andreasson et al. [18] studied the outcome differences between debulking surgery and combined CRS and HIPEC, where R1 resections were achieved in $25 \%$ of patients, and $\mathrm{R} 2$ resections achieved in $75 \%$ of patients. Multiple operations were needed in $62 \%$ for persisting tumor in this group. While the 5 year survival in the combined CRS and HIPEC was $94 \%$ with R1 resections, and $28 \%$ with $\mathrm{R} 2$ resections. Patients with tumor nodules smaller than $2.5 \mathrm{~mm}$ in diameter (CC- 0 and CC1) after cytoreduction were much more likely to survive than those with an incomplete cytoreduction [19]. Among patients who underwent CRS alone or combined with HIPEC in this study, a macroscopic complete cytoreduction (R0) was reached in $76.4 \%$ of patients. The relation between $\mathrm{R}$ score and survival in our study was $61.5 \%$ in patients with $\mathrm{R} 1 \mathrm{re}$ section however these results were not significant.

All available studies regarding the role of systemic chemotherapy are retrospective and limited to unresectable disease. Sugarbaker et al. [20] reported radiological response rate of $44 \%$ progression free survival of 6.9 months, and $50 \%$ progressive disease. Sugarbaker et al. [21] analyzed the role of FOLFOX4 neoadjuvant chemotherapy, and reported $29 \%$ objective response rate; but there was also 50\% progression. However neoadjuvant chemotherapy is considered a poor prognostic factor regarding progression free survival and overall survival [22]. In this study there was no significant relation between treatment with chemotherapy and the overall survival. 
Cytoreductive surgery combined with HIPEC is a quite complicated operation that carries significant rates of morbidity and mortality. This risk is acceptable in such lethal disease. And so many studies reported variable rates of operative and post-operative complications. In a systematic review on the efficacy of cytoreductive surgery and perioperative intraperitoneal chemotherapy for PMP, reported overall morbidity rate varied from $33 \%$ to $56 \%$. And overall mortality rate ranged from $0 \%$ to $18 \%$. With mean hospital stay ranged from 26 to 29 days, and median hospital stay from 16 to 21 days [23].

The incidence of complications depended more on the extent of the disease, number of peritonectomy procedures, and time required to complete the cytoreduction, rather than as direct association with the intraperitoneal chemotherapy administration [24].

Smeek et al. [25] studied the morbidity and mortality in patients with PMP treated with CRS and HIPEC, and reported treatment related morbidity of 54\%, mainly surgery related and reported treatment related mortality of $11 \%$. Baratti et al. [26] evaluated the outcome of combined CRS and HIPEC, and reported grade 3 - 5 surgical complications occurred in $18.7 \%$ of patients following surgery, while operative mortality occurred in one patient.

In this study we reported intraoperative complications developed in $29 \%$ of patients. Operative mortality was $22.2 \%$. Among patients who had any trial for CRS and HIPEC, operative complications were significantly related to post-operative mortality, and overall survival. Comparing these results with the previously mentioned studies results showed that we have an average operative complications rate. However small number of patients stands against reporting significant results.

A two-step approach may be used by doing HIPEC five days after CRS as a trial to decrease operative morbidity. The delayed HIPEC offers a chance to avoid long operation time for the patient and the surgeon, and also a chance to diagnose complications related to CRS as anastomotic leak that can be treated before HIPEC. However the long ICU stay and the cost of two operations are disadvantages limiting its use. Moreover adhesions and fibrin barrier limits the chemotherapy penetration to the tumor decreasing its cytotoxicity [27].

Survival and progression of the disease depends on multiple prognostic factors including PCI score, completeness of cytoreduction, tumor histology, prior surgical score, and previous lines of chemotherapy. These results showed no difference in short term survival between serial debulking and HIPEC. This finding suggested that CRS and HIPEC do not cause dramatic improvement in survival within the first 5 years. However in the long-term the survival benefits become more apparent, as the natural history of the disease is moderately slow. However survival is not the only aspect that matters. As number of reoperations were higher for the debulking era group than those for HIPEC era group (1.6 vs. 0.8). Andreasson et al. 2012 [18] reported 5 year overall survival of $74 \%$ for HIPEC compared to $40 \%$ for debulking surgery. 
Development of the combined CRS and HIPEC, as a treatment, has significantly improved outcomes of PMP, regarding survival and disease free survival. The outcome of treatment depends on numerous factors that affect prognosis, and lack of homogeneity between centers makes a comparison of results across various studies difficult, this makes prediction of factors for short-term outcomes unclear.

In a multicenter study, Elias et al. [8] analyzed data of 301 patients with PMP treated with surgery and intraperitoneal chemotherapy. The overall survival was $89 \%$ at 1 year, $84 \%$ at 3 years, $72 \%$ at 5 years and $54 \%$ at 10 years. Disease free survival was $56 \%$ at 5 years. Grade 3 to 4 complications occurred in $40 \%$ of patients that required reoperation in $17.5 \%$ of them. Chua et al. [7] in a multi institutional study including more than 2000 patients, reported 3 years survival of $80 \%, 5$ years survival of $74 \%, 10$ years survival of $64 \%$ and 15 years survival of $59 \%$. Their study also reported progression free survival of 8.2 years with median survival of 16.3 years. In a series of 465 patients in the UK, an overall survival rate at 5 years of $87 \%$, and $74 \%$ at 10 years [28]. In a study to evaluate the outcome and recurrence after CRS and HIPEC in 512 patients with PMP, Lord et al. [5] reported $26 \%$ of patients who had complete cytoreduction then developed recurrence during the follow up period, $25 \%$ of those recurred patents underwent redo surgery with complete tumor removal in $57 \%$ of them. The disease free survival at 5 years was $90 \%$ in no recurrence patients, $79 \%$ in patients who had recurrence and redo surgery, and $64 \%$ in those who had recurrence without redo surgery. Smeenk et al. [6] studied patients with PMP and reported a 3 year survival of $70.9 \%$ and 5 year. Baratti et al. [26] evaluated the outcome of combined CRS and HIPEC, and reported an overall survival of $79 \%$ at 5 years, and disease free survival of $42 \%$ at 5 years.

The follow up period in our study was quite short. The mean follow up period was 22.9 months, along with the small number of patients, were two factors against reporting significant results regarding treatment outcome. The learning curve in different centers regarding PMP treatment is extremely long as it depends on various factors including; the procedure itself, surgeons and the way they achieve their experience and proficiency which depends mainly on external observer based fellowships, and number of principal surgeons per center 'centers with more than 3 principal surgeons were at higher risk of their performance getting worse over time [29]. To acquire optimal experts, at least 100 procedures per center and 96 procedures per surgeon are necessary. This raised a fact that, the greater the institutional specialization in treatment of PMP, the lower the rate of early failure [29].

A limitation of this study remains the small number of patients that affected the significance of our final results and conclusion. So the need for more centralization through continuous cooperation between other centers, by referring PMP patients to more expert and specialized centers. Along with continuous collection of data over a long period of years is considered necessary for stronger and significant conclusions. 
In order to identify the most effective follow up regimen, research is ongoing to identify patients at higher risk of recurrence and the risk factors. However currently, recurrence is diagnosed when there is marked rise in tumor markers, or evidence of relapse with CT [6]. The diagnosis of progressive disease is not always easy, as after extensive surgery CT scans are usually difficult to interpret due to post-surgical changes. So it is useful to make a baseline CT scan 3 months after primary surgery, and compare further CT scans [6]. CT has low sensitivity to detect tumor deposits less than $5 \mathrm{~mm}$, tumors located on lesser omentum, root of mesentery, left hemi-diaphragm, and bowel wall due to the use of oral contrast. MRI is more sensitive to detect these tumors. However availability of high resolution $\mathrm{CT}$ and its rapid technique makes it the primary imaging modality compared to MRI that has long scanning time, high cost and more dependence on radiologist experience [30]. PET/CT has better diagnostic accuracy compared to MRI, as well as its coverage of the whole body giving information about possible metastasis; (PET/CT vs MRI) sensitivity 93/87\%, specificity 96/92\% [30].

The post-operative follow up protocol we use is to do CT scan and tumor markers every 3 months in year one, then every 6 months in year 2 and 3, then annually, with PET/CT once per year. Patients, with mucinous neoplasms, should be followed for years to detect PMP in early stage. A minimal follow up for 5 years consisting of physical examination, tumor markers, CEA and CA 19-9 and CT scan once a year or when indicated is reasonable. This is because of the long latency time and indolent tumor behavior [6].

\section{Conclusion}

The outcome of PMP treatment process is extremely variable. Combined CRS and HIPEC is considered the best therapeutic approach for patients with PMP. Surgical experience combined with proper patient selection have to be built up together to improve the outcome. That could only be achieved through more centralization of patients' treatment in specialized units or center.

\section{References}

[1] Andreasson, H., Lorant, T., Pahlman, L., Graf, W. and Mahteme, H. (2014) Cytoreductive Surgery plus Perioperative Intraperitoneal Chemotherapy in Pseudomyxoma Peritonei: Aspects of the Learning Curve. European Journal of Surgical Oncology, 40, 930-936. https://doi.org/10.1016/j.ejso.2014.03.001

[2] McBride, K., McFadden, D. and Osler, T. (2013) Improved Survival of Patients with Pseudomyxoma Peritonei Receiving Intraperitoneal Chemotherapy with Cytoreductive Surgery: A Systematic Review and Meta-Analysis. Journal of Surgical Research, 183, 246-252. https://doi.org/10.1016/j.jss.2012.12.040

[3] Alves, S., Mohamed, F., Yadegarfar, G., Youssef, H. and Moran, B.J. (2010) Prospective Longitudinal Study of Quality of Life Following Cytoreductive Surgery and Intraperitoneal Chemotherapy for Pseudomyxoma Peritonei. European Journal of Surgical Oncology, 36, 1156-1161. https://doi.org/10.1016/j.ejso.2010.09.004

[4] Chua, T.C., Yan, T.D., Smigielski, M.E., Zhu, K.J., Ng, K.M., Zhao, J. and Morris, D.L. (2009) Long-Term Survival in Patients with Pseudomyxoma Peritonei Treated 
with Cytoreductive Surgery and Perioperative Intraperitoneal Chemotherapy: 10 Years of Experience from a Single Institution. Annals of Surgical Oncology, 16, 1903-1911. https://doi.org/10.1245/s10434-009-0341-8

[5] Lord, A.C., Shihab, O., Chandrakumaran, K., Mohamed, F., Cecil, T.D. and Moran, B.J. (2014) Recurrence and Outcome after Complete Tumour Removal and Hyperthermic Intraperitoneal Chemotherapy in 512 Patients with Pseudomyxoma Peritonei from Perforated Appendiceal Mucinous Tumors. European Journal of Surgical Oncology, 41, 396-399. https://doi.org/10.1016/j.ejso.2014.08.476

[6] Smeenk, R.M., Verwaal, V.J., Antonini, N. and Zoetmulder, F.A. (2007) Survival Analysis of Pseudomyxoma Peritonei Patients Treated by Cytoreductive Surgery and Hyperthermic Intraperitoneal Chemotherapy. Annals of Surgery, 245, 104-109. https://doi.org/10.1097/01.sla.0000231705.40081.1a

[7] Chua, T.C., Moran, B.J., Sugarbaker, P.H., Levine, E.A., Glehen, O., Gilly, F.N., Baratti, D., Deraco, M., Elias, D., Sardi, A., Liauw, W., Yan, T.D., Barrios, P., Gómez Portilla, A., de Hingh, I.H., Ceelen, W.P., Pelz, J.O., Piso, P., González-Moreno, S., Van Der Speeten, K. and Morris, D.L. (2012) Early- and Long-Term Outcome Data of Patients with Pseudomyxoma Peritonei from Appendiceal Origin Treated by a Strategy of Cytoreductive Surgery and Hyperthermic Intraperitoneal Chemotherapy. Journal of Clinical Oncology, 30, 2449-2456. https://doi.org/10.1200/JCO.2011.39.7166

[8] Elias, D., Gilly, F., Boutitie, F., Quenet, F., Bereder, J.M., Mansvelt, B., Lorimier, G., Dubè, P. and Glehen, O. (2010) Peritoneal Colorectal Carcinomatosis Treated with Surgery and Perioperative Intraperitoneal Chemotherapy: Retrospective Analysis of 523 Patients from a Multicentric French Study. Journal of Clinical Oncology, 28, 63-68. https://doi.org/10.1200/JCO.2009.23.9285

[9] Taflampas, P., Dayal, S., Chandrakumaran, K., Mohamed, F., Cecil, T.D. and Moran, B.J. (2014) Pre-Operative Tumour Marker Status Predicts Recurrence and Survival after Complete Cytoreduction and Hyperthermic Intraperitoneal Chemotherapy for Appendiceal Pseudomyxoma Peritonei: Analysis of 519 Patients. European Journal of Surgical Oncology, 40, 515-520.

https://doi.org/10.1016/j.ejso.2013.12.021

[10] Panarelli, N.C. and Yantiss, R.K. (2011) Mucinous Neoplasms of the Appendix and Peritoneum. Archives of Pathology \& Laboratory Medicine, 135, 1261-1268. https://doi.org/10.5858/arpa.2011-0034-RA

[11] Sugarbaker, P.H. (2005) Cytoreductive Surgery and Perioperative Intraperitoneal Chemotherapy: A New Standard of Care for Appendiceal Mucinous Tumors with Peritoneal Dissemination. Clinics in Colon and Rectal Surgery, 18, 204-214. https://doi.org/10.1055/s-2005-916281

[12] Sugarbaker, P.H. (2013) Pseudomyxoma Peritonei and Peritoneal Metastases from Appendiceal Malignancy. In: Sugarbaker, P.H., Ed., Cytoreductive Surgery and Perioperative Chemotherapy for Peritoneal Surface Malignancy. Textbook and Video Atlas, 3, 57-75.

[13] Sugarbaker, P.H. and Esquivel, J. (2000) Clinical Presentation of the Pseudomyxoma Peritonei Syndrome. British Journal of Surgery, 87, 1414-1418. https://doi.org/10.1046/j.1365-2168.2000.01553.x

[14] de Bree, E., Koops, W., Kroger, R., van Ruth, S., Verwaal, V.J. and Zoetmulder, F.A. (2006) Preoperative Computed Tomography and Selection of Patients with Colorectal Peritoneal Carcinomatosis for Cytoreductive Surgery and Hyperthermic Intraperitoneal Chemotherapy. European Journal of Surgical Oncology, 32, 65-71. https://doi.org/10.1016/j.ejso.2005.09.016 
[15] Menassel, B., Duclos, A., Passot, G., Dohan, A., Payet, C., Isaac, S., Valette, P.J., Glehen, O. and Rousset, P. (2016) Preoperative CT and MRI Prediction of Non-Resectability in Patients Treated for Pseudomyxoma Peritonei from Mucinous Appendiceal Neoplasms. European Journal of Surgical Oncology, 42, 558-566. https://doi.org/10.1016/j.ejso.2016.01.005

[16] Elias, D., Lefevre, J.H., Chevalier, J., Brouquet, A., Marchal, F., Classe, J.M., Ferron, G., Guilloit, J.M., Meeus, P., Goéré, D. and Bonastre, J. (2009) Complete Cytoreductive Surgery plus Intraperitoneal Chemohyperthermia with Oxaliplatin for Peritoneal Carcinomatosis of Colorectal Origin. Journal of Clinical Oncology, 27, 681-685. https://doi.org/10.1200/JCO.2008.19.7160

[17] Jarvinen, P., Ristimaki, A., Kantonen, J. and Lepisto, A. (2013) Feasibility of Radical Cytoreductive Surgery and Hyperthermic Intraperitoneal Chemotherapy for Pseudomyxoma Peritonei of Appendicular Origin. Scandinavian Journal of Surgery, 102, 145-151. https://doi.org/10.1177/1457496913490463

[18] Andreasson, H., Graf, W., Nygren, P., Glimelius, B. and Mahteme, H. (2012) Outcome Differences between Debulking Surgery and Cytoreductive Surgery in $\mathrm{Pa}$ tients with Pseudomyxoma Peritonei. European Journal of Surgical Oncology, 38, 962-968. https://doi.org/10.1016/j.ejso.2012.07.009

[19] Harmon, R.L. and Sugarbaker, P.H. (2005) Prognostic Indicators in Peritoneal Carcinomatosis from Gastrointestinal Cancer. International Seminars in Surgical Oncology, 2, 3. https://doi.org/10.1186/1477-7800-2-3

[20] Sugarbaker, P. and Chang, D. (1999) Results of Treatment of 385 Patients with Peritoneal Surface Spread of Appendiceal Malignancy. Annals of Surgical Oncology, 6, 727-731. https://doi.org/10.1007/s10434-999-0727-7

[21] Sugarbaker, P.H., Bijelic, L., Chang, D. and Yoo, D. (2010) Neoadjuvant FOLFOX Chemotherapy in 34 Consecutive Patients with Mucinous Peritoneal Carcinomatosis of Appendiceal Origin. Journal of Surgical Oncology, 102, 576-581. https://doi.org/10.1002/jso.21679

[22] Chen, C.-F., Huang, C.-J., Kang, W.-Y. and Hsieh, J.-S. (2008) Experience with Adjuvant Chemotherapy for Pseudomyxoma Peritonei Secondary to Mucinous Adenocarcinoma of the Appendix with Oxaliplatin/Fluorouracil/Leucovorin (FOLFOX4). World Journal of Surgical Oncology, 6, 118. https://doi.org/10.1186/1477-7819-6-118

[23] Yan, T.D., Black, D., Savady, R. and Sugarbaker, P.H. (2007) A Systematic Review on the Efficacy of Cytoreductive Surgery and Perioperative Intraperitoneal Chemotherapy for Pseudomyxoma Peritonei. Annals of Surgical Oncology, 14, 484-492. https://doi.org/10.1245/s10434-006-9182-X

[24] Stephens, A.D., Alderman, R., Chang, D., Edwards, G.D., Esquivel, J., Sebbag, G., Steves, M.A. and Sugarbaker, P.H. (1999) Morbidity and Mortality of 200 Treatments with Cytoreductive Surgery and Hyperthermic Intraoperative Intraperitoneal Chemotherapy Using the Coliseum Technique. Annals of Surgical Oncology, 6, 790-796. https://doi.org/10.1007/s10434-999-0790-0

[25] Smeenk, R.M., Verwaal, V.J. and Zoetmulder, F.A.N. (2006) Toxicity and Mortality of Cytoreduction and Intraoperative Hyperthermic Intraperitoneal Chemotherapy in Pseudomyxoma Peritonei-A Report of 103 Procedures. European Journal of Surgical Oncology, 32, 186-190. https://doi.org/10.1016/j.ejso.2005.08.009

[26] Baratti, D., Kusamura, S., Nonaka, D., Langer, M., Andreola, S., Favaro, M., Gavazzi, C., Laterza, B. and Deraco, M. (2008) Pseudomyxoma Peritonei: Clinical Pathological and Biological Prognostic Factors in Patients Treated with Cytoreductive 
Surgery and Hyperthermic Intraperitoneal Chemotherapy (HIPEC). Annals of Surgical Oncology, 15, 526-534. https://doi.org/10.1245/s10434-007-9691-2

[27] Mohr, Z., Hirche, C., Liebeskind, U., Rau, B.M. and Hünerbein, M. (2011) Feasibility of Delayed Hyperthermic Intraperitoneal Chemotherapy in Case of Unforeseen Complications. European Surgical Research, 47, 19-25.

https://doi.org/10.1159/000327397

[28] Youssef, H., Newman, C., Chandrakumaran, K., Mohamed, F., Cecil, T.D. and Moran, B.J. (2011) Operative Findings, Early Complications, and Long-Term Survival in 456 Patients with Pseudomyxoma Peritonei Syndrome of Appendiceal Origin. Diseases of the Colon \& Rectum, 54, 293-299. https://doi.org/10.1007/DCR.0b013e318202f026

[29] Kusamura, S., Moran, B.J., Sugarbaker, P.H., Levine, E.A., Elias, D., Baratti, D., Morris, D.L., Sardi, A., Glehen, O., Deraco, M., Peritoneal Surface Oncology Group International (PSOGI), et al. (2014) Multicentre Study of the Learning Curve and Surgical Performance of Cytoreductive Surgery with Intraperitoneal Chemotherapy for Pseudomyxoma Peritonei. British Journal of Surgery, 101, 1758-1765. https://doi.org/10.1002/bjs.9674

[30] Krishnamurthy, S. and Balasubramaniam, R. (2016) Role of Imaging in Peritoneal Surface Malignancies. Indian Journal of Surgical Oncology, 7, 441-452.

https://doi.org/10.1007/s13193-016-0539-8 